\section{Conflictos de intereses en nefrología}

\author{
SOFÍA P. SALAS ${ }^{1,2}$, ANTONIO VUKUSICH ${ }^{1,3}$, MARÍA ISABEL CATONI ${ }^{1,4}$, \\ ANDRÉS VALDIVIESO ${ }^{1,5}$, EMILIO ROESSLER ${ }^{1,6}$
}

\section{Conflicts of interest in nephrology}

Since doctors disposed of effective tools to serve their patients, they had to worry about the proper management of available resources and how to deal with the relationship with the industry that provides such resources. In this relationship, health professionals may be involved in conflicts of interest that they need to acknowledge and learn how to handle. This article discusses the conflicts of interest in nephrology. Its objectives are to identify those areas where such conflicts could occur; to help to solve them, always considering the best interest of patients; and to help health workers to keep in mind that they have to preserve their autonomy and professional integrity. Conflicts of interest of professionals in the renal area and related scientific societies, with the industry producing equipment, supplies and drugs are reviewed. Dichotomy, payment for referral, self-referral of patients and incentives for cost control are analyzed. Finally, recommendations to help preserve a good practice in nephrology are made.

(Rev Med Chile 2016; 144: 1053-1058)

Key words: Conflict of interest; Ethics; Nephrology; Physician Self-Referral; Renal Dialysis.
${ }^{1}$ Comité de Ética Sociedad

Chilena de Nefrología.

${ }^{2}$ Facultad de Medicina,

Universidad Diego Portales.

Santiago, Chile.

${ }^{3}$ Facultad de Medicina,

Universidad de los Andes.

Santiago, Chile.

${ }^{4}$ Escuela de Enfermería, Pontificia Universidad Católica de Chile.

Santiago, Chile.

${ }^{5}$ Facultad de Medicina, Pontificia Universidad Católica de Chile.

Santiago, Chile.

${ }^{6}$ Facultad de Medicina, Clínica

Alemana-Universidad del

Desarrollo. Santiago, Chile.

Conflictos de intereses: Ninguno que declarar. Este trabajo no obtuvo financiamiento.

Recibido el 11 abril de 2016, aceptado el 5 julio de 2016.

Correspondencia a

Dra. Sofía P. Salas

Ejército 260; Santiago, Chile.

Fono: (562) 22676.2829

sofia.salas@udp.cl
$\mathrm{D}$ esde que los médicos dispusieron de herramientas eficaces para atender a sus pacientes, han debido preocuparse del manejo de los escasos recursos disponibles y de cómo enfrentar la relación con la industria que provee esos recursos. Por una parte, los médicos deben aplicar un criterio de justicia en la distribución de los recursos, que suele entrar en conflicto con el principio de beneficencia. Por otra parte, en sus relaciones con la industria pueden verse involucrados en conflictos de intereses que, aunque indeseables, pueden ser inevitables, por lo que es necesario aprender cómo abordarlos según las buenas prácticas en medicina ${ }^{1}$.

La hemodiálisis aguda se inició en Chile en el año 1957 en el Hospital San Francisco de Borja, mientras que el primer centro de hemodiálisis crónica (CHC) privado se creó en $1974^{2}$; no obstante, el acceso al tratamiento siguió siendo muy limitado. Por estos motivos, los nefrólogos debieron integrar comisiones institucionales que resolvían qué pacientes urémicos eran elegidos para ingresar a un programa de hemodiálisis crónica (HDC) y así continuar viviendo. En la década siguiente, el Estado estimuló el emprendimiento de médicos nefrólogos y otros profesionales de la salud, además de inversionistas, para la creación de $\mathrm{CHC}$ privados a lo largo del país. Se produjo entonces un crecimiento exponencial de la oferta de HDC que permitió el acceso universal a esta terapia; un enorme logro del país y de los profesionales del área renal.

Este avance, que podríamos denominar técnico-clínico, no ha estado exento de problemas ético-clínicos. En efecto, este año el Comité de Ética de la Sociedad Chilena de Nefrología dio a conocerlos resultados de un estudio en el cual se consultó la opinión de médicos y enfermeras/os que trabajan en $\mathrm{CHC}$ respecto de eventuales dilemas éticos percibidos durante su trabajo ${ }^{3}$. Casi un 
15\% de los encuestados señaló que podrían tener conflictos de intereses personales al momento de decidir la pertinencia de la HDC, cifra que se duplica cuando se pregunta por la posibilidad de estos conflictos en otros miembros del equipo. El beneficio económico fue el principal conflicto de intereses señalado.

Este artículo analiza los conflictos de intereses en nefrología, incluyendo los de la HDC. Sus objetivos son identificar aquellas áreas donde podrían ocurrir conflictos de intereses; contribuir a que los profesionales de la salud pongan atención al deber de preservar su autonomía e integridad profesional; y ayudar a resolver dichos conflictos, considerando siempre el mejor interés de los pacientes.

\section{Concepto de conflicto de intereses}

La Asociación Médica Mundial (AMM) define "conflicto de intereses de la práctica médica" como aquella situación donde el correcto juicio profesional requerido para el cuidado directo de un paciente puede estar influenciado indebidamente por intereses secundarios" ". De manera similar, la Asociación de Sociedades Científicas-Médicas de Chile considera que existe un conflicto de intereses cuando un juicio o acción que debería estar determinado por un valor primario, definido por razones profesionales o éticas, podría estar o aparecer influido por un segundo interés ${ }^{5}$. A su vez, uno de los principios del profesionalismo médico es considerar siempre que el interés del paciente está por sobre el del médico ${ }^{6}$. El Código de Ética del Colegio Médico de Chile tiene una sección específica dedicada a las relaciones con las empresas de productos clínicos y farmacéuticos, instando a mantener una relación de independencia con las empresas proveedoras de productos o servicios médicos, sin "jamás perseguir la obtención de beneficios personales" (Art. 54) ${ }^{7}$.

\section{Conflictos de intereses en la relación de los profesionales de la salud con la industria}

La AMM reconoce que la relación entre médicos e industria ha contribuido al desarrollo de nuevos métodos diagnósticos, terapias innovadoras y dispositivos médicos que pueden implicar un gran desarrollo para la medicina y mejoría en la calidad de vida ${ }^{8}$. Sin embargo, la existencia de conflictos de intereses puede afectar la autonomía y reputación de los profesionales de la salud y el cuidado de los pacientes. La evidencia empírica muestra que luego de reunirse con representantes de una determinada industria, los médicos cambian sus prácticas de prescripción y solicitan, con mayor frecuencia, incorporar fármacos o insumos de esa industria a su hospital; asimismo, si las actividades de educación continua son financiadas por la industria, aumenta la prescripción de los productos de la compañía que las financia' ${ }^{9}$. Si bien este tema es menos visible y ha sido menos tratado en el área de enfermería, también está presente, ya que estos profesionales tienen poder de decisión en la compra de determinados aparatos, dispositivos o insumos de uso clínico. La asistencia a cursos o congresos de enfermería financiados por la industria pudiera ser un factor de influencia indirecta en la toma de decisiones de compra de insumos en un CHC.

El Código de Ética del Colegio Médico contiene recomendaciones específicas para hacer frente a los conflictos de intereses ${ }^{7}$. En su Art. 55 señala que: "El médico sólo podrá aceptar donaciones modestas o invitaciones a reuniones o congresos, proporcionadas por empresas de productos clínicos o farmacéuticos, cuando aquéllas no limiten o coarten su independencia profesional", mientras que el Art. 56 estipula que: "Infringe la ética profesional el médico que aceptare financiamiento, total o parcial, de actividades ajenas a la profesión médica, por parte de empresas de productos clínicos o farmacéuticos, tales como viajes con fines turísticos, u otras similares". Por su parte, la industria también considera una falta a la ética el entregar beneficios, independiente del tipo y monto, a cambio de prescribir sus productos o prometer fidelidad a éstos ${ }^{10}$.

Cuando un profesional prescribe un fármaco o un procedimiento -diagnóstico o terapéuticosabiendo que es más conveniente para sí mismo que para su paciente, se aleja del correcto juicio clínico y del debido cuidado de los pacientes, vulnerando varios principios éticos: el principio de beneficencia, si deja de prescribir lo mejor para su paciente; el de autonomía, si no se presentan al paciente todas las opciones disponibles; el de no maleficencia, si aumenta el riesgo de fracaso del tratamiento o de eventos adversos; y el de justicia, 
tanto para el paciente como para las instituciones, si aumentan innecesariamente los costos ${ }^{11}$.

\section{Conflictos de intereses en la relación de las sociedades científicas con la industria}

Las sociedades científicas tienen un papel fundamental en definir los estándares de cuidado en salud, promover la educación continua, elaborar guías clínicas, publicar en revistas especializadas e implementar normas deontológicas ${ }^{12}$. Adicionalmente, algunas financian trabajos de investigación, mantienen registros de datos médicos y algunos de sus miembros integran comités que determinan las prestaciones que se otorgarán a una determinada población. Por otra parte, también reciben una importante ayuda económica de la industria, con la cual desarrollan congresos y actividades de educación continua. La industria suele financiar, además, la asistencia de profesionales de la salud a dichas actividades. En consecuencia, las sociedades médicas ciertamente están expuestas a conflictos de intereses con la industria. Estos deben ser anticipados y regulados, aunque ello afecte el financiamiento de algunas actividades. Sociedades médicas extranjeras han implementado una estricta política al respecto, que incluye la declaración de potenciales conflictos de intereses entre sus miembros, especialmente sus directivos; la separación física -en un congreso- entre el área de promociones y el resto del evento, de modo que no sea obligatorio transitar por dicha zona entre otras medidas de transparencia ${ }^{13}$. Luego de evaluar la experiencia señalan que, contra lo esperado, no hubo una caída significativa en el financiamiento de las actividades educacionales, lo que puede ser un ejemplo a imitar de forma gradual y adaptándolo a nuestra realidad ${ }^{12}$.

La Academia de Medicina de Chile también ha efectuado recomendaciones respecto de cómo enfrentar los potenciales conflictos de intereses, tanto a nivel de presentaciones a congresos como en la redacción de guías clínicas. Recomienda que en las presentaciones públicas, congresos científicos, conferencias y todo tipo de reuniones profesionales o académicas, los médicos participantes hagan trasparentes todas sus relaciones personales con la industria, de modo de poder identificar y sopesar la presencia o influencia de eventuales conflictos de intereses. Asimismo, deben realizar esta declaración aquellos que contribuyen a "redactar guías clínicas médicas o a integrar comités profesionales destinados a tomar decisiones administrativas, tales como compra de insumos, fármacos, técnicas diagnósticas, equipos $\mathrm{u}$ otros productos propios de la industria, y para fijar normas clínicas o consensos de diagnóstico o tratamiento". Se considera imprescindible "que el control académico del programa científico permanezca siempre en manos de las instituciones académicas organizadoras, asegurando la independencia científica de los contenidos de las conferencias, cursos y talleres de educación médica" ${ }^{14}$. Por lo tanto, es recomendable que nefrólogos y enfermeras/os dedicadas a nefrología, trasplante o diálisis realicen obligatoriamente una declaración de posibles conflictos de intereses cada vez que efectúen una presentación o redacten una publicación. Una forma de conciliar la necesidad de financiar la formación continua y los intereses de la industria es que los fondos se entreguen siempre a las instituciones científicas $o$ académicas y no a personas individuales. Las instituciones deben implementar formas de recibir y emplear dichos recursos que impidan los vínculos personales entre los profesionales y la industria. Por ejemplo, se pueden instituir convenios o fondos de donación de carácter central que sean recibidos por las instituciones académicas o científicas y luego empleados en forma transparente para financiar actividades de formación continua.

\section{Conflictos de intereses en nefrología}

La nefrología nació como una disciplina derivada de la medicina interna, que se dedicaba a la fisiología, fisiopatología, anatomía patológica y laboratorio renal. Posteriormente, los tratamientos de reemplazo renal como peritoneodiálisis y hemodiálisis aguda y luego crónica empezaron a ocupar una parte importante del quehacer nefrológico. Los avances tecnológicos y el financiamiento permitieron extender su uso a $\mathrm{CHC}$ ambulatorios, mejorando la sobrevida y la calidad de vida de cientos de miles de pacientes con enfermedad renal crónica terminal. Sin embargo, estos importantes beneficios pueden entrar en conflicto con intereses económicos, produciendo desconfianza y desprestigio de la especialidad. 


\section{Dicotomía, pago por derivación y autoreferencia de pacientes}

El concepto de dicotomía en el ámbito médico se refiere a la repartición de honorarios entre médicos u otros profesionales que previamente han acordado derivarse pacientes ${ }^{15}$. De acuerdo al Código de Ética del Colegio Médico de Chile, "falta a la ética el médico que cobre o pague a otro profesional para la derivación de pacientes, o reciba comisiones por realizar exámenes complementarios"7. Con esta práctica no sólo se desvirtúa el significado del honorario médico, pudiendo aumentar innecesariamente el costo de la prestación, sino que además se pierde la libertad del paciente de elegir al profesional o centro de salud que desee.

Uno de los temas que merece analizarse cuidadosamente es cuando el médico clínico deriva a sus pacientes a un $\mathrm{CHC}$ de su propiedad, donde tiene intereses económicos o recibe un pago por derivación ${ }^{16}$. En Estados Unidos de Norteamérica, la ley federal prohíbe a los médicos derivar a pacientes del seguro Medicare a entidades en las cuales tienen intereses financieros, en lo que ha sido llamado "autoreferencia"17,18. La evidencia empírica ha mostrado que cuando los criterios de derivación están influidos por un interés financiero se produce un mayor uso de procedimientos o mayores costos para el usuario que cuando los enfermos se derivan a proveedores independientes, como ha sido documentado para servicios de imágenes y anatomía patológica ${ }^{18}$.

Esta relación financiera -estudiada principalmente en los Estados Unidos de Norteamérica- entre los CHC y el médico que indica el tratamiento, y que debe evaluar la posibilidad de suspenderlo por considerarlo desproporcionado, o que debe promover en sus pacientes el trasplante renal, es compleja. Ciertamente, en casos de dicotomía evidente -con reparto de honorarios por enviar/ recibir enfermos- el profesional de la salud pierde su autonomía y tiene un claro conflicto de intereses; pero no toda autoderivación tiene como objetivo obtener ingresos adicionales, ni se la puede considerar dentro del concepto dicotomía. En los comienzos de la HDC chilena, especialmente en las regiones, un grupo de nefrólogos fundaba un $\mathrm{CHC}$ y acudían a él pacientes que no tenían otro sitio donde dializarse porque el servicio público no les proporcionaba la terapia. También se producían derivaciones a determinados $\mathrm{CHC}$, porque los profesionales estaban genuinamente interesados en continuar asistiendo a sus pacientes, en esta nueva etapa de la enfermedad, o porque tenían confianza en la calidad de su trabajo. Hoy día existe una amplia oferta de CHC que compiten entre sí, algunos están agrupados en sociedades, otros pertenecen a empresas transnacionales y existe la posibilidad real de derivación de pacientes sólo por intereses económicos. Cuando el médico aparece como cuidador del paciente $y$, al mismo tiempo, debe ser gestor de recursos porque él mismo -o en sociedad con otros- es dueño de un hospital o centro de salud, la ganancia financiera por derivar puede influir de manera inapropiada en el juicio clínico, con los riesgos de sobreutilización de los servicios en los cuales el médico tiene inversiones, aumento de costos y eventualmente disminución de la calidad del cuidado, puesto que pueden haber incentivos económicos inadecuados al ahorro ${ }^{19}$. Una de las estrategias para disminuir el impacto de la autoreferencia es transparentar las relaciones comerciales, incluyendo la obligación de reportar al sistema de salud y también a los pacientes la existencia de estos acuerdos comerciales. Igualmente importantes son los controles que debe realizar la autoridad sanitaria para garantizar un cuidado homogéneo y de calidad en todos los CHC.

Es comprensible, entonces, la inquietud por la posibilidad de que los nefrólogos influyan en los pacientes para que se cambien de médico o de CHC, en lo que ha sido llamado como "solicitación de pacientes". Todas estas prácticas cuestionan el profesionalismo médico ${ }^{20}$. Para que los pacientes puedan tomar decisiones informadas, se requiere revelar de manera honesta y objetiva toda evidencia respecto de la calidad de los $\mathrm{CHC}$ donde derivan a sus pacientes y también cualquier interés financiero ${ }^{20}$. A su vez, la AMM ha sido enfática en señalar que la autoderivación o el recibir una compensación económica por derivar pacientes a un determinado sistema de salud, son prácticas inaceptables ${ }^{4}$. Por extensión, estas prácticas son también inaceptables para otros profesionales de la salud. Existe el deber ético de preservar la autonomía e integridad profesional, tanto entre los dueños de CHC que pudiesen proponer estas prácticas, como entre los profesionales que las acepten. En Chile, las comisiones derivadoras de pacientes a HDC en cada hospital base debieran velar porque la indicación sea apropiada y que hayan sido informadas todas las opciones de tratamiento, incluido el tratamiento con diálisis peritoneal, 
hemodiálisis, tratamiento médico conservador o paliativo y trasplante renal, al paciente, a su familia o a ambos. Asimismo, es necesario para mantener la transparencia del sistema, que las comisiones derivadoras publiquen periódicamente el número de pacientes enviados a cada CHC y los criterios considerados en la decisión.

En HDC existen, además, potenciales conflictos de intereses cuando no hay plena independencia entre el profesional a cargo del $\mathrm{CHC}$ y la industria que vende los insumos, ya sea maquinarias, sueros, filtros, etc. En este contexto, la Academia Chilena de Medicina recomienda que "la relación entre los médicos y la industria se caracterice siempre y en toda circunstancia por una total transparencia, que respalde la confianza del público, de los pacientes y de todos los actores involucrados en este proceso" ${ }^{\prime \prime}$.

\section{Incentivos al control de costos}

Un problema ético frecuente en HDC se refiere al control de costos que pudiese poner en peligro el bienestar de los pacientes ${ }^{21}$. En principio, la reducción de costos manteniendo la calidad técnica permite un mayor acceso de pacientes al procedimiento, y es consustancial al ambiente de libre competencia; pero la falta de controles de calidad (por ejemplo re-utilizando filtros más allá de los recomendado), puede poner en peligro la seguridad de los pacientes. Los profesionales deben tomar conciencia de que toda presión sobre ellos mismos o sobre el personal técnico para reducir los costos que pudiese afectar la calidad y seguridad de la atención es inaceptable, ya que los pone en la disyuntiva de perjudicar a los pacientes para mantener su trabajo o mejorar sus ingresos.

\section{Conclusiones y recomendaciones}

El manejo de los conflictos de intereses en la práctica médica y de las profesiones de la salud ha sido, y seguirá siendo, un tema complejo del cual no están ajenos los profesionales de CHC. Para enfrentarlos proponemos las siguientes sugerencias y recomendaciones:

1. Más allá del saber técnico-biológico, debe instalarse como eje de la enseñanza en las profesiones de la salud el poner siempre, en primer lugar, el bienestar del paciente, procurando una atención segura y de calidad objetiva.
2. Se deben promover conductas correctas y virtuosas, tanto en la educación médica como en el ejercicio profesional.

3. Debe profundizarse el entrenamiento práctico en bioética desde el pre-grado y como parte del proceso de formación continua de médicos nefrólogos y enfermeras. Dado que la responsabilidad ética es siempre personal, es importante la temprana toma de conciencia sobre la importancia de preservar siempre la autonomía e integridad profesional.

4. Debe transparentarse, pública y claramente, en las presentaciones académicas o en los trabajos enviados a congresos la existencia o no de conflictos de intereses.

5. Debe promoverse una política de responsabilidad respecto de la oportunidad, pertinencia y calidad de la HDC.

6. Sugerimos evitar toda relación directa entre la industria y los profesionales o estudiantes de carreras de la salud que pueda producir conflictos de intereses.

7. Toda vez que sea posible, sugerimos tener una relación con la industria de tipo institucional, a través de sociedades científicas o centros médicos y centros académicos, para que éstos administren fondos para educación continua bajo reglas claras y de manera transparente.

8. Los médicos que tienen intereses financieros en $\mathrm{CHC}$ deben transparentarlos y permitir a los pacientes elegir entre su CHC y otras opciones de igual calidad.

\section{Referencias}

1. Besio M. Sobre el acto médico. Revista Conamed 2006; 11(8): 20-5.

2. Valdivieso A. Historia de la Diálisis, en Historia de la Sociedad Chilena de Nefrología, M. Alvo, Editor. Imprenta Salesianos SA, Santiago 2004. pp 207-87.

3. Vukusich A, Catoni MI, Salas SP, Valdivieso A, Browne F, Roessler E. Problemas ético-clínicos en hemodiálisis crónica: percepción de médicos y enfermeras. Rev Med Chile 2016; 144: 14-21.

4. World Medical Association, Statement on conflict of Interest. Adoptado por la 60a Asamblea General, Nueva Delhi, India, octubre 2009.

5. Asociación de sociedades científicas-médicas de Chile. [Recommendations to avoid conflicts of interest with the pharmaceutical companies]. Rev Med Chile 2005; 133: 607-8. 
6. Abim Foundation ABoIM, Acp-Asim Foundation ACoP-ASoIM, European Federation of Internal Medicine. Medical professionalism in the new millennium: a physician charter. Ann Intern Med 2002; 136(3): 243-6.

7. Código de Ética del Colegio Médico de Chile AG, 2011.

8. World Medical Association, Statement concerning the relationship between physicians and commercial enterprises. Adoptado por la 55a Asamblea General de la AMM, Tokyo, Japón, octubre 2004 y modificada en la 60ª Asamblea General de la AMM, Nueva Delhi, India, octubre 2009.

9. Wazana A. Physicians and the pharmaceutical industry: is a gift ever just a gift? JAMA 2000; 283(3): 373-80.

10. Pharmaceutical Research and Manufacturers of America, PhRMA Code on Interactions with Healthcare Professionals, 2008.

11. Salas SP, Osorio FM, Vial CP, Rehbein VA, Salas AC, Beca IJ. [Conflicts of interest in clinical practice. Ethical analysis of some relationships with the pharmaceutical industry]. Rev Med Chile 2006; 134 (12): 1576-82.

12. Rothman DJ, McDonald WJ, Berkowitz CD, Chimonas SC, DeAngelis CD, Hale RW, et al. Professional medical associations and their relationships with industry: a proposal for controlling conflict of interest. JAMA 2009; 301 (13): 1367-72.

13. Schofferman JA, Eskay-Auerbach ML, Sawyer LS, Herring SA, Arnold PM, Muehlbauer EJ. Conflict of interest and professional medical associations: the North American Spine Society experience. Spine J 2013; 13(8): 974-9.

14. Academia Chilena de Medicina, Documento de la Academia Chilena de Medicina sobre las relaciones entre los médicos y la industria proveedora de salud, 2014: Boletín de la Academia Chilena de Medicina p. 329-32.

15. Rosas Lavado H. Dicotomía: Patología de la relación médico paciente. Revista Peruana de Radiología 2007; 11: 37-8.

16. Mitchell JM, Sunshine JH. Consequences of physicians' ownership of health care facilities-joint ventures in radiation therapy. N Engl J Med 1992; 327 (21): 1497-501.

17. Council on Ethical and Judicial Affairs AMA. Conflicts of interest. Physician ownership of medical facilities. JAMA 1992; 267 (17): 2366-9.

18. Mitchell JM. Linkages between utilization of prostate surgical pathology services and physician self-referral. Medicare Medicaid Res Rev 2012; 2(3).

19. Cole CM. Physician-owned hospitals and self-referral. Virtual Mentor 2013; 15(2): 150-5.

20. Ozar DT, Kristensen C, Fadem SZ, Blaser R, Singer D, Moss AH. Nephrologists' professional ethics in dialysis practices. Clin J Am Soc Nephrol 2013; 8 (5): 840-4.

21. Ulrich CM, Taylor C, Soeken K, O'Donnell P, Farrar A, Danis M, et al. Everyday ethics: ethical issues and stress in nursing practice. J Adv Nurs 2010; 66 (11): 2510-9. 